\title{
ASPECTOS EPIDEMIOLÓGICOS DOS ACIDENTES POR ANIMAIS PEÇONHENTOS OCORRIDOS NO MUNICÍPIO DE CACOAL-RO, NO PERÍODO DE 2007-2016
}

\author{
EPIDEMIOLOGICAL ASPECTS OF ACCIDENTS BY SUBSTANTIAL ANIMALS OCCURRED \\ IN THE CACOAL-RO MUNICIPALITY IN THE PERIOD 2007-2016
}

\author{
Gleison Faria ${ }^{a^{*}}$, Angela Antunes de Morais Lima ${ }^{b^{*}}$
}

gleisonfaria@hotmail.coma , angel.antunesml@gmail.com ${ }^{\mathrm{b}}$

FACIMED

Data de submissão: 28/01/2019

Data de Aceite: 21/10/2019

\section{RESUMO}

\begin{abstract}
Animais peçonhentos são todos animais que possuem veneno e causa efeitos colaterais ao organismo do ser humano. Objetivo: Analisar incidência de casos de acidentes de animais peçonhentos notificados em Cacoal - RO no período de 2007 a 2016. Metodologia: estudo documental, transversal, descritiva com abordagens quantitativas com uso de um questionário semiestruturado de 23 perguntas de múltiplas escolhas extraídos da ficha de notificação do ministério da saúde, teve a aprovação do CEP, e dispensa do termo livre esclarecido. Obteve-se resultados de 315 notificações sendo 72,69\% no sexo masculino e 27,31\% feminino, correspondendo $46,34 \%$ na faixa etária de 30 a 59 anos, tendo maior ocorrência na zona rural do município com $82,85 \%$ e zona urbana $17,15 \%$. Em relação aos acidentes notificados $65,07 \%$ por serpentes os quais $49,52 \%$ por gênero Brotópico espécie jararaca, 23,17\% escorpiões sendo todos ignorados o gênero e espécie na hora da notificação, 7,61\% aranhas sendo ignorado maior parte das notificações o gênero e espécie do animal, 4,12\% outros tipos de animais peçonhentos. Cerca de 39,68 tiveram atendimento de 1 a 3 horas. O membro mais acometido foram os pés com 37,14\% devido a falta do uso de EPI. Conclui-se - A incidência dos casos de acidentes por animais peçonhentos ocorrido no município pode ser considerada alto, sendo em média 35 casos/ano. Ocorre em muitas localidades devido ao período chuvoso, devido ao aumento da água dos rios que leva os animais para pastagens planas mais próximas as residências, sendo assim, os animais se sente ameaço e acaba atacando.
\end{abstract}

Palavras-chave: Animais venenosos; vigilância epidemiológica; epidemiologia

\section{ABSTRACT}

Venomous animals are all animals that have poison and cause side effects to the body of the human being. Objective - To analyze the incidence of accidents of venomous animals reported in Cacoal - RO from 2007 to 2016. Methodology - descriptive, cross - sectional documentary study with quantitative approaches using a semistructured questionnaire of 23 multiple choice questions extracted from the notification of the ministry of health, had the approval of the CEP, and exemption from the free term clarified. The results of 315 notifications were $72.69 \%$ in males and $27.31 \%$ in females, corresponding to $46.34 \%$ in the age range of 30 to 59 years, with a greater occurrence in the rural area of the municipality with $82.85 \%$ and urban area $17.15 \%$. In relation to the reported incidents $65.07 \%$ by snakes which $49.52 \%$ by genus Brotópico species jararaca, $23.17 \%$ scorpions being all ignored the genus and species at the time of notification, $7.61 \%$ spiders being ignored most of the notifications the gender and species of the animal, $4.12 \%$ other types of venomous animals. About 39.68 were attended for 1 to 3 hours. The most affected limb was the feet with $37.14 \%$ due to lack of use of EPI. It is concluded - The incidence of accidents by venomous animals occurred in the municipality can be considered high, with an average of 35 cases / year. It occurs in many localities due to the rainy season, due to the increase of river water that takes the animals to flat pastures closer to the residences, so the animals feel threatened and end up attacking.

Keywords: Poisonous animals; epidemiological surveillance; epidemiology 


\section{Introdução}

Os acidentes acometidos por animais peçonhentos no Brasil, envolvem em maior quantidade a população da zona rural. Os trabalhadores do sexo masculino são os mais notificados e são incluído como notificações de doenças tropicais negligenciadas ${ }^{12}$. Animais peçonhentos segundo Brasil ${ }^{24}$ são todos aqueles animais que possuem veneno e que de alguma forma causa efeitos colaterais ao organismo do ser humano. As espécies que serão abordadas nesta pesquisa são, serpentes, escorpiões e aranhas ${ }^{14,17,24,25}$.

No Brasil, entre 2010 e 2014 foram notificados mais de 691.307 acidentes por animais peçonhentos no Sistema de Informação de Agravos de Notificação - SINAN, dos quais 1.282 evoluíram para óbito ${ }^{18,22,23}$. O munícipio de Cacoal-RO fica localizado a $480 \mathrm{~km}$ da capital de Rondônia (Porto Velho), e vem tendo um aumento significativo com o passar dos anos, com maior incidência na zona rural ${ }^{13}$.

O aumento das chuvas e alagamentos da encosta dos rios e igarapés localizado no município propiciam a invasão e manifestação das espécies peçonhentas a procura de abrigos devido ao grande volume de agua ${ }^{29}$. Observa-se que o índice de desmatamento e queimadas é grande, ou seja, o homem e o principal culpado por destruir o seu habitat natural fazendo com que as espécies de animais possam se aproximar cada vez mais das residências, tornando o contato animal/homem mais frequente ${ }^{3}$. Embora que esses animais não só estão ocasionando vítimas na zona rural como também na zona urbana, pois através do período chuvoso as serpentes através das enchentes se aproximam das residências e procuram seu habitat em locais úmidos e sujos e quando se sente ameaçadas elas atacam ${ }^{1,31}$.

Dentre os animais peçonhentos existentes, os mais comuns das espécies peçonhentas que são as serpentes, aranhas e escorpiões, devido surgirem em lugares inesperados nas áreas urbanas e serem as espécies mais notificados pelos órgãos do município $^{22,31}$.

Esta pesquisa justifica-se, pois trata de uma situação que ocorre a nível nacional, trata-se de um estudo sobre a quantidade de casos que acometem a população do município de CacoalRO, principalmente na região da zona rural, onde lavradores são os que mais sofrem com esse tipo de ataque, principalmente do gênero Bothrops (jararaca) e Lachesis (surucucu pico de jaca) ${ }^{32,33}$.

O objetivo geral da pesquisa foi analisar a incidência de casos de acidentes de animais peçonhentos notificados em Cacoal - RO no período de 2007 a 2016.

\section{Metodologia}

O presente trabalho apresentou-se como sendo documental retrospectivo, transversal, descritiva com abordagem quantitativa ${ }^{24}$. A pesquisa foi realizada no município de Cacoal-RO, teve como instrumento de coleta de dados um questionário semiestruturado de 23 perguntas de múltiplas escolhas que foram extraídos da própria ficha de notificação da doença abordando critérios de inclusão com as seguintes variáveis: Identificar o perfil do paciente quanto a faixa etária, sexo, cor, escolaridade, e zona de ocorrência, antecedentes epidemiológicos, dados clínicos, dados do acidente e evolução do caso, a mesma, com intuito de direcionamento da coleta de dados, as quais foram extraídas as informações secundarias do banco de dados do sistema de informação de agravos de notificação - SINAN que foram fornecidas pela secretaria municipal de saúde do município setor de vigilância em saúde pelo servidor responsável, disponibilizados para os pesquisadores em forma de planilhas no Microsoft Excel ${ }^{\oplus 5-7,18,21}$.

Antes de iniciar a pesquisa foi encaminhado para o comitê de ética e pesquisa (CEP), da Faculdade de ciências biológicas de Cacoal-RO, por meio da sua aprovação recebeu o número do parecer consubstanciado no 2.064.193.

A pesquisa teve início nos meses de maio e junho de 2017, e obteve uma amostra de 315 notificações da doença. Como critério de exclusão, foram descartados todos os casos não pertencentes ao município de Cacoal, ou que não estava incluído dentro o período estipulado, antes de receber os dados foram encaminhados via e-mail para a vigilância em saúde exatamente os dados necessários para a pesquisa (itens 10$14,18,20,38-43,45-47,49-55,57)$ da ficha de 
notificação da doença), sendo assim, facilitando para o responsável do setor de vigilância em saúde a filtração do banco de dados do SINAN, os quais após foram disponibilizados de acordo como solicitado para os pesquisadores, não ouve custos nem remuneração pelos dados obtidos e nem exposição da integridade dos pacientes notificados, não foi necessário o uso do termo de consentimento livre esclarecido - TCLE pois os dados foram secundários sendo assim solicitado a dispensa do mesmo ao $\mathrm{CEP}^{5-7,18,21}$.

\section{Resultados}

Os resultados apresentados no decorrer deste trabalho cientifico, ocorreu na região do município de Cacoal-RO, onde através de aulas no decorrer do semestre acadêmico, foi abordado tema através do corpo docente, informado o alto índice de casos de acidentes de animais peçonhentos na região, com base nesta informações surgiu o interesse da pesquisa com dados extraídos de 2007 a 2016 com amostra de 315 casos notificados no SINAN de dos tipos serpentes, aranha e escorpião, sendo zona rural $82,85 \%$ e zona urbana $17,14 \%$, sendo que a população total do município e de estimativa de 87.877 habitantes ${ }^{13}$.
DADOS DEMOGRÁFICOS DAS VÍTIMAS DE ANIMAIS PEÇONHENTOS OCORRIDOS NO MUNICÍPIO DE CACOAL-RO, PERÍODO DE 2007 A 2016

Com base nos dados extraídos na tabela 1 , notase que o maior índice de acidentes acometidos por animais peçonhentos no ano de 2007 a 2016 foram a idade de 30-59 anos (46,34\%), em seguida 15-29 anos (25,07\%), 7-14 anos (12,38\%), 60 anos acima e 0-6 anos (10,79\%). A idade de 30-59 com 146 vítimas conforme dados da tabela acima, observa que $72,69 \%$ destas vítimas são do sexo masculino, pois são o que tem maior contato com o campo no qual é o habitat natural dos animais peçonhentos e $27,31 \%$ são mulheres.

Observa que o grau de escolaridade das vítimas são: $23,49 \%$ tem de 1 à $4^{\text {a }}$ série, $14,92 \%$ tem de $5^{\mathrm{a}}$ à $8^{\mathrm{a}}$ série, $12,69 \%$ tem o ensino fundamental completo, $8,25 \%$ são analfabetos, $6,98 \%$ tem a $4^{\text {a }}$ série completa, $5,07 \%$ tem o ensino médio completo, $1,90 \%$ tem nível superior incompleto, $1,90 \%$ tem nível superior completo e 19,36 das vítimas não informaram no momento da coleta dos dados pela ficha de notificação do SINAN.

Tabela 1- Perfil demográfico dos pacientes, Cacoal-RO, 2007 - 2016.

\begin{tabular}{|c|c|c|c|}
\hline \multicolumn{2}{|c|}{ DADOS } & $\mathbf{N}$ & $\%$ \\
\hline \multirow{2}{*}{ Sexo } & Masculino & 229 & 72,69 \\
\hline & Feminino & 86 & 27,31 \\
\hline \multicolumn{2}{|c|}{ TOTAL } & 315 & $100 \%$ \\
\hline \multirow{5}{*}{ Faixa etária } & $0 \vdash 6$ & 17 & 5,3 \\
\hline & 7 -14 & 39 & 12,4 \\
\hline & $15 \mid-29$ & 79 & 25,08 \\
\hline & $30 \vdash 59$ & 146 & 46,34 \\
\hline & 60 anos acima & 34 & 10,79 \\
\hline \multicolumn{2}{|c|}{ TOTAL } & 315 & $100 \%$ \\
\hline
\end{tabular}




\begin{tabular}{|c|c|c|c|}
\hline \multicolumn{2}{|c|}{ DADOS } & $\mathbf{N}^{\mathbf{0}}$ & $\%$ \\
\hline \multirow{10}{*}{ Escolaridade } & Analfabeto & 26 & 8,25 \\
\hline & $1^{\mathrm{a}}$ à $4^{\mathrm{a}}$ Serie & 74 & 23,49 \\
\hline & $4^{\mathrm{a}}$ serie completo & 22 & 6,98 \\
\hline & $5^{\mathrm{a}}$ à $8^{\mathrm{a}}$ serie inc. & 47 & 14,92 \\
\hline & Ensino Fund. Completo & 40 & 12,69 \\
\hline & Ensino Médio Completo & 18 & 5,71 \\
\hline & Ensino Médio Incompleto & 16 & 5,07 \\
\hline & Ensino Sup. Incompleto & 6 & 1,90 \\
\hline & Ensino Sup. Completo & 6 & 1,90 \\
\hline & Ignorado & 61 & 19,36 \\
\hline \multicolumn{2}{|c|}{ TOTAL } & 315 & $100 \%$ \\
\hline \multirow{2}{*}{$\begin{array}{l}\text { Local de } \\
\text { Ocorrência do acidente }\end{array}$} & Zona rural & 261 & 82,85 \\
\hline & Zona urbana & 54 & 17,15 \\
\hline \multicolumn{2}{|c|}{ TOTAL } & 315 & $100 \%$ \\
\hline
\end{tabular}

Fonte: SINAN/SEMUSA/MS, 2007-2016, Cacoal - RO

TIPOS DE ANIMAIS PEÇONHENTOS DA REGIÃO DE CACOAL-RO

Os gêneros de serpentes mais comum na região de Cacoal-RO, são Botrópicos, Laquetico, Elapídico, Crotálico, as aranhas são os gêneros Foneustrimo e Loxoscelismo e os escorpiões todos os casos foram ignorados e não informado a espécie.

Os acidentes foram informados dentre os anos de 2007 a 2016 no município de Cacoal - RO, zona rural e zona urbana, a identificação dos números de casos dos acidentes informa que 65,07\% dos acidentes informados no sistema foram acometidos por serpentes, $7,61 \%$ acometidos por aranhas e $23,17 \%$ por escorpiões e $4,12 \%$ acometidos por outros animais peçonhentos que não serão identificados devido o objetivo central ser os gêneros especificados acima. Dentre os $65,07 \%$ das vítimas informadas por serpentes, pode-se classificar segundo dados obtidos pelo SINAN que $49,52 \%$ delas foram acometidos pelo gênero botrópicos (jararaca), 0,33\% foram acometidos pelo gênero crotálico (cascavel), $0,63 \%$ foram acometidos pelo gênero elapídico (coral verdadeira), 4,12\% foram acometidos pelo gênero laquetico (surucucu pico de jaca), 4,76\% não peçonhentos e $5,71 \%$ dos casos não foram informados e considerados como ignorados.

Dentro dos 7,61\% dos acidentes informados por Aranhas, pode-se classificar que 2,22\% dos casos foram acometidos pelo gênero foneustrimo (aranha armadeira), 1,26\% foram acometidos pelo gênero loxoscelismo (aranha marrom) e 4,12\% dos casos acometidos foram ignorados por não ser identificados o gênero da peçonha. Dentro os $23,17 \%$ dos acidentes acometidos pelos animais peçonhentos do tipo escorpião, $100 \%$ dos casos, foram classificados pela ficha de notificação do SINAN, como ignorados por não ter sido classificado o gênero e nem espécie do animal. 
Tabela 2 - Tipo de acidente/ tipo de animal - Cacoal-RO, 2007 - 2016.

\begin{tabular}{|c|c|c|c|}
\hline ANIMAL & GÊNERO & $\mathbf{N}^{0}$ & $\%$ \\
\hline \multirow{6}{*}{ Serpentes } & Botrópico & 156 & 49,52 \\
\hline & Crotálico & 1 & 0,33 \\
\hline & Elapídico & 2 & 0,66 \\
\hline & Laquetico & 13 & 4,12 \\
\hline & Não peçonhenta & 15 & 4,76 \\
\hline & Ignorado & 18 & 5,71 \\
\hline \multicolumn{2}{|c|}{ TOTAL } & 205 & 65,07 \\
\hline \multirow{3}{*}{ Aranhas } & Foneustrimo & 7 & 2,22 \\
\hline & Loxoscelismo & 4 & 1,26 \\
\hline & Ignorado & 13 & 4,12 \\
\hline \multicolumn{2}{|c|}{ TOTAL } & 24 & 7,61 \\
\hline Escorpião & $\begin{array}{l}\text { Não informada gênero } \\
\text { /espécie }\end{array}$ & 73 & 23,17 \\
\hline Outros & Outros tipos & 13 & 4,12 \\
\hline \multicolumn{2}{|c|}{ TOTAL } & 315 & $100 \%$ \\
\hline
\end{tabular}

Fonte: SINAN/SEMUSA/MS, 2007-2016, Cacoal - RO

TEMPO DECORRIDO DO ATENDIMENTO MÉDICO AS VÍTIMAS DE ACIDENTES POR ANIMAIS PEÇONHENTOS DENTRO DO MUNICÍPIO DE CACOAL-RO

Foram identificados que $22,22 \%$ das vítimas procuraram atendimento médico entre 0 a 1 hora, $39,68 \%$ das vítimas procuraram atendimentos médico entre 1 a 3 horas, $21,26 \%$ das vítimas procuraram atendimentos médico entre 3 a 6 horas, $8,88 \%$ das vítimas procuraram atendimentos entre 6 a 12 horas, $1,26 \%$ das vítimas procuraram atendimento entre 12 a 24 horas e $6,66 \%$ das vítimas procuraram atendimento acima de 24 horas, todos os dados informados foram óbitos pelo SINAN. 
Tabela 3 - Tempo decorrido do Atendimento- Cacoal-RO, 2007 - 2016.

\begin{tabular}{c|c|c|c}
\hline \multicolumn{2}{c|}{ DADOS } & $\mathbf{N}^{\mathbf{0}}$ & \% \\
\hline \multirow{4}{*}{$\begin{array}{c}\text { Tempo Decorrido/ } \\
\text { Atendimento }\end{array}$} & $0 \vdash 1$ & 70 & 22,22 \\
\cline { 2 - 4 } & $1-3$ & 125 & 39,68 \\
\cline { 2 - 4 } & $3 \vdash 6$ & 67 & 21,26 \\
\cline { 2 - 4 } & $6 \vdash 12$ & 28 & 8,88 \\
\cline { 2 - 4 } & $12-24$ & 4 & 1,26 \\
\hline \multirow{2}{*}{ TOTAL horas acima } & 21 & 6,66 \\
\hline
\end{tabular}

Fonte: SINAN/SEMUSA/MS, 2007-2016, Cacoal - RO

LOCAIS DE OCORRÊNCIAS DO ACIDENTE OCASIONADO PELO ANIMAL PEÇONHENTO

Os locais serão classificados como cabeça, braço, antebraço, mão, dedo da mão, tronco, coxa, perna, pé, dedo do pé e os casos que foram ignorados ou não identificados na hora da coleta dos dados pela ficha de notificação no Sistema de
Informação de Agravos de Notificação (SINAN). Com $37,14 \%$ o local dos pés, com $23,49 \%$ as pernas, com $13,01 \%$ as mãos, com $9,52 \%$ os dedos das mãos, com $5,71 \%$ os dedos dos pés, com $2,53 \%$ as coxas, com $2,22 \%$ a cabeça, braço e o antebraço, com $1,26 \%$ os dedos da mão e $0,63 \%$ dos locais foram ignorados ou não informados na ficha de notificação do SINAN.

Tabela 4 - Local da Picada - Cacoal-RO, 2007 - 2016.

\begin{tabular}{|c|c|c|c|}
\hline \multicolumn{2}{|c|}{ DADOS } & $\mathbf{N}$ & $\%$ \\
\hline \multirow{11}{*}{ Local da Picada } & Cabeça & 7 & 2,22 \\
\hline & Braço & 7 & 2,22 \\
\hline & Antebraço & 7 & 2,22 \\
\hline & Mão & 41 & 13,01 \\
\hline & Dedo da mão & 30 & 9,52 \\
\hline & Tronco & 4 & 1,26 \\
\hline & Coxa & 8 & 2,53 \\
\hline & Perna & 74 & 23,49 \\
\hline & Pé & 117 & 37,14 \\
\hline & Dedo do pé & 18 & 5,71 \\
\hline & Ignorado & 2 & 0,63 \\
\hline \multicolumn{2}{|c|}{ TOTAL } & 315 & $100 \%$ \\
\hline
\end{tabular}

Fonte: SINAN/SEMUSA/MS, 2007-2016, Cacoal - RO 
MANIFESTAÇÕES PROVOCADAS PELA AÇÃO DO VENENO DA PEÇONHA NO ORGANISMO DAS VÍTIMAS

Das vítimas que foram picadas $84,44 \%$ por algum dos tipos de animais peçonhentos (serpente, aranha e escorpião) sofreram algum tipo de manifestação local, e 15,56\% não tiveram nenhum tipo de manifestação ou não foi informado na ficha de notificação do SINAN. Os tipos de manifestações que os 315 pacientes notificados no SINAN apresentaram:

Dos pacientes que apresentaram manifestações locais $88,88 \%$ dos pacientes apresentaram dor no local da picada, $74,60 \%$ dos pacientes notificados apresentaram edema no local da picada, $12,06 \% \%$ dos pacientes notificados apresentaram equimose, $8,25 \%$ dos pacientes notificados apresentaram necrose no local da picada e $7,61 \% \%$ dos pacientes apresentaram outros tipos de sintomas como (dormência, eritema, hematomas, rouquidão, bolhas, focos infecciosos, linfonodomegalias inguinais, cianose, parestesia e hiperemia).

Conforme apresentado na tabela 12,38\% dos pacientes que foram picados por algum dos tipos de animais peçonhentos (serpente, aranha e escorpião) sofreram algum tipo de manifestação sistêmica, e 87,61\% não tiveram nenhum tipo de manifestação ou não foi informado na ficha de notificação do SINAN (tabela 5).
Dentre os tipos de manifestações sistêmicas ocasionados pelos animais peçonhentos do tipo (serpente, aranha e escorpião) que foram notificados através da ficha de notificação do SINAN conforme item 43 da ficha de notificação do SINAN, foram informados os tipos de manifestações sistêmicas apresentadas nos pacientes que sofreram acidentes com as espécies de animais citadas acima, as manifestações informadas são: neuroparalíticas (ptose palpebral, turvação visual), miolíticas/hemolíticas (mialgia, anemia, urina escura), hemorrágicas (gengivorragia, outros sangramentos), renais (oligúria/anúria), vagais (vômitos, diarreias) e outras manifestações especificadas.

Dos pacientes que sofreram manifestações sistêmicas após a inoculação do veneno, 6,03\% apresentaram vagais (vômitos, diarreias), $3.80 \%$ dos pacientes apresentaram neuroparalíticas (ptose palpebral, turvação visual), $0,31 \%$ dos pacientes apresentaram miolíticas/hemolíticas (mialgia, anemia, urina escura), 2,22\% dos pacientes apresentaram alterações renais (oligúria/anúria) e dentre outros 6,98\% apresentaram sintomas como (febre, sangramento, cefaleia, dispneia, vertigem, náuseas, sincope e adenomegalia), nenhum paciente apresentou hemorragias.

Tabela 5 - Manifestações locais / Se manifestações locais - Manifestações Sistêmica - Se Manifestações sistêmicas Cacoal-RO, 2007 - 2016.

\begin{tabular}{c|c|c|c}
\hline \multicolumn{2}{c|}{ DADOS } & N & \% \\
\hline \multirow{3}{*}{\begin{tabular}{c} 
Manifestações locais \\
\cline { 2 - 4 }
\end{tabular}} & Sim & 266 & 84,44 \\
\cline { 2 - 4 } & Não & 49 & 15,56 \\
\hline \multirow{2}{*}{$\begin{array}{c}\text { TOTAL manifestações } \\
\text { Locais }\end{array}$} & Dor & 315 & $100 \%$ \\
\cline { 2 - 4 } & Edema & 280 & 88,88 \\
\cline { 2 - 4 } & Equimose & 235 & 74,60 \\
\cline { 2 - 4 } & Necrose & 26 & 12,06 \\
\cline { 2 - 5 } & Outros & 24 & 8,25 \\
\hline
\end{tabular}




\begin{tabular}{c|c|c|c}
\hline \multirow{2}{*}{$\begin{array}{c}\text { Manifestações } \\
\text { Sistêmica }\end{array}$} & Sim & 39 & 12,38 \\
\cline { 2 - 4 } & Não & 276 & 87,61 \\
\hline \multirow{2}{*}{ TOTAL } & 315 & $100 \%$ \\
\hline \multirow{4}{*}{$\begin{array}{c}\text { Se manifestações } \\
\text { Sistêmicas }\end{array}$} & Neuroparalíticas & 12 & 3,80 \\
\cline { 2 - 4 } & Miolíticas & 1 & 0,31 \\
\cline { 2 - 4 } & Hemorrágicas & 0 & 0 \\
\cline { 2 - 4 } & Renais & 7 & 2,22 \\
\cline { 2 - 4 } & Vagais & 22 & 6,03 \\
\cline { 2 - 4 } & Outros & 19 & 6,98 \\
\hline
\end{tabular}

Fonte: SINAN/SEMUSA/MS, 2007-2016, Cacoal - RO

\section{DISCUSSÃO}

A Portaria no 1.138/GM/MS, de 23 de maio de 2014 define as ações e os serviços de saúde voltados para vigilância, prevenção e controle de zoonoses e acidentes causados por animais peçonhentos e venenosos, de relevância para a saúde pública ${ }^{15}$. Casos são esses, que ocorrer constantemente com a população, tanta zona rural quanto zona urbana. As vítimas do sexo masculino correspondem a maioria por ter a participação no trabalho agrícola em geral e a dona casa que corresponde o sexo feminino auxilia consideravelmente o homem nas atividades do campo que incluir o plantio e colheita de alimentos para sobrevivência ${ }^{26}$.

Conforme dado BRASIL ${ }^{16,23,24}$ foram notificados de 2007 a 2015 no estado de Rondônia 4.312 acidentes acometidos por serpentes, 1.136 acidentes acometidos por escorpião e 723 acidentes acometidos por aranhas. Dados que corresponde a $4,75 \%$ das notificações de serpentes na cidade de Cacoal-RO, 6,42\% das notificações de escorpião na cidade de Cacoal-RO e 3,31\% das notificações de aranha na cidade de Cacoal - $\mathrm{RO}^{27,28}$.
Os membros inferiores e superiores são áreas mais atingidas, sendo assim sempre e recomendado que as pessoas que trabalham no campo com lavouras ou pastagens usem botas, perneiras, luvas e outros EPI'S, onde possam prevenir muitos desses acidentes ${ }^{20}$. Dentre os registros notificados no SINAN de 2007 a 2016, foram identificados que na zona rural nos anos de 2012 teve uma incidência de $11,74 \%$ dos acidentes e em 2016 correspondeu a $10,47 \%$ dos acidentes. E entre os registros de 2007 a 2016 foram identificados que na zona urbana o ano de 2012 correspondeu a $2,22 \%$ e 2013 com a mesma taxa de $2,22 \%{ }^{30}$.

É possível que a incidência de registro possa ter ocorrido pelas fortes chuvas no ano de 2012 que atingiu o município de Cacoal- $\mathrm{RO}$, onde promoveu uma grande enchente que alagou diversas áreas da cidade $^{1,4,27,28}$. Tendo em vista que com o aumento das chuvas, e o aumento dos volumes dos rios, aumenta o número de predadores no campo e consequentemente também das serpentes, aranhas e escorpião ${ }^{31}$.

Das vítimas que foram notificadas algumas tiveram complicações conforme o item 53 da ficha de notificação, os quais foram informados 
que $1,90 \%$ dos pacientes apresentaram infecções secundárias, $1,58 \%$ dos pacientes apresentaram necrose extensa, $0,63 \%$ dos pacientes apresentaram síndrome compartimental e déficit funcional, nenhum dos pacientes necessitou fazer amputação de membros. Dos pacientes informados apresenta $60 \%$ das notificações as quais sofreram acidentes e tiveram complicações ou sintomas leves, $34,9 \%$ dos casos foram considerados quadro moderado e $5,07 \%$ dos casos foram considerados quadro grave, e nenhum caso foi ignorado ${ }^{10}$.

O tratamento com soroterapia correspondeu a $49,52 \%$ dos casos a vítimas acometidas pela picada de serpentes do gênero botrópicos, $23,17 \%$ pela picada de escorpião do gênero/ espécie não identificada, mais que provavelmente seja as espécies mais comuns na região Tityus obscurus (escorpião preto $)^{15,23,25,28}$. Na análise dos dados lançado no SINAN, não foi informado se o paciente utilizou outros meios de tratamento como: corticoides, profilaxia do tétano, antibióticos e anti-histamínico, pois não foi analisado nenhum prontuário medico das vítimas. Embora na leitura de outros artigos, observa-se que a maior parte dos acidentes acometidos por qualquer espécie de animais peçonhentos é ocorrido no trabalho9. $\mathrm{Na}$ pesquisa realizada em Cacoal - RO (zona rural e zona urbana) observa - se que cerca de $69,20 \% \%$ dos acidentes acometidos pelos animais peçonhentos não são ocorridos em locais de trabalho e $30,80 \%$ dos acidentes ocorre nos locais de trabalho das vítimas, como plantio, colheitas das safras e aumento da vegetação. Foi informado melhora ou cura em $98,73 \%$ dos pacientes e entre orestante foram informados $1,27 \%$ de pacientes os quais evoluíram a óbito, talvez por ter procurado atendimento tardio, ou por não saber identificar o animal corretamente para tomar a devida conduta terapêutica ${ }^{22,34}$.

Os óbitos referentes aos acidentes pelos animais peçonhentos ocorreram em 2011, 2012 e 2014, o tempo decorrido até o atendimento foi de 1 a 3 horas e acima de 24 horas, e todos pacientes morava na zona rural do município. Todos os 4 óbitos que foram notificados através da ficha de notificação no Sistema de Informação de Agravos de Notificação (SINAN), foram ocasionados pela picada do escorpião da espécie Tityus obscurus (escorpião preto), que a espécie mais comum na região norte ${ }^{2,23,25}$ na análise dos dados, verificou-se que somente um paciente foi informado corretamente a conduta terapêutica, sendo administrado a quantidade 20 ampolas da sorologia antiescorpiônico (SAEs), os outros 3 pacientes não foram informados se usaram ou não o tratamento com a sorologia e qual conduta foi tomada. Os dois pacientes que antes de evoluir para óbito em 2011 sobreviveram após a picado do animal peçonhento um com 12 dias e outros com 31 dias, os de 2012 sobreviveu 20 dias e o de 2014 também sobreviveu 20 dias após a inoculação do veneno da peçonha. É possível que isto tenha ocorrido pela administração de dose inadequada de soro ou pela não identificação correta do tipo do animal (SINAN) ${ }^{22,34}$.

A conduta errada realizada por pessoas simples sem a devida experiência ou conhecimento cientifica faz com que na hora do desespero acabam aplicando ervas, fazendo uso de torniquete, colocando outros tipos de substancias nolocal da picada, neste caso perdem muito tempo, ocasionando agravo e dificultando o diagnóstico dos médicos nos prontos atendimentos do município de Cacoal- $\mathrm{RO}^{30}$.

O conhecimento e algo fundamental para se atender as vítimas referente a esse tipo de acidente, saber identificar a espécie da peçonha e iniciar a terapêutica no menor tempo e imprescindível a saúde desse paciente ${ }^{37}$.

\section{Conclusão}

Conclui-se que a incidência dos casos de acidentes por animais peçonhentos dentro do município pode ser considerada alto, ocorrendo em média 35 casos/ano, isso ocorre em muitas localidades devido ao período chuvoso, ocorrendo o aumento da água dos rios e levando os animais para pastagens planas mais próximas as residências, sendo assim, os animais se sente ameaço e acaba atacando para se proteger.

Muito das complicações ocorridas, se refere ao tempo do atendimento, ou seja, as vítimas que residem na zona rural são maioria classe baixa e não tem conduções próprias para esta socorrendo o acidentado, embora muito das vezes tendo que 
recorrer a terceiros, tendo assim uma demora até a realização dos primeiros socorros a unidade de saúde mais próxima.

Ao passar dos anos os acidentes com animais peçonhentos vem tendo uma incidência significativa, ou seja, alguns anos aumenta, outros diminuem. O acometimento desses acidentes na zona urbana, evidencia também pela não realização da manutenção das redes de saneamento básico, que passam nas cidades onde muito desses animais se alojam e acabam indo para os quintais das casas, e quando se sentem ameaçados atacam. Observa-se que a taxa de mortalidade relacionada a esse tipo de acidentes na cidade de Cacoal -RO foi de $0,04 / 1000$ habitantes.

A profilaxia indicada para esse tipo de acidente, seria criar treinamento para os agentes comunitários de saúde inclusive da zona rural referente ao uso correto de EPI'S e também a limpeza ao redores das casas, e o que fazer e a quem chamar em caso de um acidente, realizar palestras educativas nas escolas, treinamentos dos enfermeiros para o correto preenchimento das fichas de notificações para que não deixar nenhum campo em branco, pois um dos campos bem importante na maioria dos casos não são informados, como foi a quantidade de sorologia utilizada.

Fator bem importante na ficha de notificação do paciente e o tipo de notificação que informa se o acidente está ou não relacionado no trabalho, pois considera-se todo morador da zona rural trabalhador, ou seja, são agricultores e pecuarista, entretanto a maior parte dos pacientes notificados corresponde a zona urbana e somente $30,80 \%$ dos casos foram notificados como acidente de trabalho.

\section{Agradecimentos}

Agradecimentos a docentes e amigos que contribuíram com apoio a execução da pesquisa.

\section{REFERENCIAS}

1. Agencia de notícias \& Publicidade. Alerta sobre o aumento de animais peçonhentos em Rondônia. Rondônia: 2017 - [citado em 01 abr. 2017] - Disponível em: https://www.ariquemesonline.com.br/noticia. asp? $\operatorname{cod}=253597 \& \operatorname{codDep}=31$

2. Bochner, R, Struchiner, CJ - Epidemiologia dos acidentes ofídicos nos últimos 100 anos no Brasil: uma revisão Rio de Janeiro: 2003 - [internet] - Cad. Saúde Pública, Rio de Janeiro, 19(1):7-16

3. Casagrande, B - Caracterização do meio físico e avaliação do desmatamento no município de Cacoal RO - [Dissertação de Mestrado em Ciências Humanas]Universidade Federal de Uberlândia, Uberlândia: 2009

4. Climograma de Cacoal. Cacoal: 2017 [citado em 20 nov. 2017] Disponível em: https://www.climatempo.com.br/ noticias/88935/chuva-forte-em-cacoal-ro/

5. Comitê de ética e pesquisa. Regimento Interno - Cacoal: 2017 [internet]. Disponível em: http://facimed.edu.br/ departamentos/detalhes/cep-10

6. Comitê de ética e pesquisa. Resolução $\mathrm{n}^{\circ} 510$, de 07 de Abril de 2016 - FACIMED, Cacoal: 2017 [internet]. Disponível em: http://conselho.saude.gov. br/resolucoes/2016/reso510.pdf> e <http://facimed.edu. br/artigos/ler/resolucoes-conselho-nacional-de-saudecns-153>

7. Comitê de ética e pesquisa. Documentação Obrigatória. Cacoal: 2017 [internet] - Disponível em:http://facimed. edu.br/artigos/ler/cep-documentacao-obrigatoria-92

8. Constituição da República Federativa do Brasil de 1988 Brasília: 1988 -[citado em 15 de ago. 2017]. Disponível em:http://www.planalto.gov.br/ccivil_03/constituicao/ constitui\%C3\%A7ao.htm

9. Correia, FF et al - Perfil clínico-epidemiológico dos acidentes ofídicos ocorridos no município de Cacoal, Rondônia, Brasil, 2011 e 2015 -[ Artigo original] Revista Eletrônica FACIMEDIT, v5, n2, Ago/Dez. 2016 ISSN 1982-5285 -

10. Cotta, GA. Animais peçonhentos - $5^{\text {a }}$ edição p.11 Belo Horizonte: 2014 - [citado em 31 dez. 2017] -

Disponível em: http://www.funed.mg.gov.br/wpcontent/uploads/2010/03/cartilha.pdf

11. Fachin, O. Fundamentos de metodologia. 4. ed. São Paulo: Saraiva, 2003. $5^{\mathrm{a}}$ ediçãop. 11

12. Gutiérrez JM, Theakston, RDG., Warrell, DA. - Enfrentando o problema negligenciado da envenenamento de mordidas de cobra: a necessidade de uma parceria global, 2006, PLoS Med, v. 3, n. 6, p. e150.

13. Instituto brasileiro de geografia e estatística- Brasília: 2017 - [citado em 20 de mar. 2017] - Disponível em:http://cidades.ibge.gov.br/painel/historico. php?codmun $=110020$ 
14. Ministério da Saúde (BR). Aranhas. Brasília: Ministério da Saúde: 2016 - [citado em 03 mar. 2017]. Disponível em: http://portalsaude.saude.gov.br/index.php/o-ministerio/ principal/secretarias/svs/animais-peçonhentos-aranha

15. Ministério da Saúde (BR). Secretaria de Atenção à Saúde. Departamento de Atenção Básica. Vigilância em saúde: zoonoses. Secretaria de Atenção à Saúde, Departamento de Atenção Básica. Brasília: Ministério da Saúde: 2009[citado em 03 mar. 2017]. Disponível em: http://portal. saude.gov.br/portal/arquivos/pdf/abcad22pdf

16. Ministério da Saúde (BR). SINAN - Sistema de informações de agravos de notificações. Brasília: Ministério da Saúde: 2017 - [citado em 27 mar. 2017]. Disponível em: htpps//portalsinan.saude.gov.br/ acidente- por-animais-peçonhento

17. Ministério da Saúde (BR). Escorpião - Brasília: Ministério da Saúde: 2016 [citado 03 mar. 2017]. Disponível em: htps//portalsaude.saude.gov.br/index.php/o-ministerio/ principal/secretarias/svs/animais-peconhentosescorpioes

18. Ministério da Saúde (BR). Ficha de notificação Brasília: Ministério da Saúde: 2016 [citado 29 dez. 2017] - Disponível em: https//www.visa.goias.gov.br/post/ ver/165260/fichas-de-notific

19. Ministério da Saúde (BR). Lei no 8.080 , de 19 de setembro de 1990 Brasília: Ministério da Saúde: 2016 [citado em 15 ago. 2017] - Disponível em: http://www.planalto.gov.br/ ccivil_03/leis/18080.htm

20. Ministério da Saúde (BR). NR 4 - Serviços especializados em engenharia de segurança e em medicina do trabalho, (104.000-6) - inciso V e VII. Brasília: Ministério da Saúde: 1983- [citado em 05 abr. 2017] - Disponível em: http://sislex.previdencia.gov.br/paginas/05/mtb/4.htm

21. Ministério da Saúde (BR). Plataforma Brasil Autorização. Brasília: Ministério da Saúde: 2017 - [citado 29 mai. 2017]. Disponível em: http://aplicacao.saude.gov. br/plataformabrasil/visao/pesquisador/gerirPesquisa/ gerirPes quisaAgrupador.jsf

22. Ministério da Saúde (BR). Secretaria de Vigilância em Saúde - Acidentes por Animais Peçonhentos, Cacoal -RO, 2007-2016. Brasília: Ministério da Saúde: 2016 [citado em 15 ago. 2017] - Disponível em: http:// www.medicinanet.com.br/conteudos/biblioteca/2069/ capitulo_5_\%E2\%80\%93_acidentes_por_animais_ peconhentos.htm

23. Ministério da Saúde (BR). Secretaria de Vigilância em Saúde. Departamento de Vigilância Epidemiológica. Doenças infecciosas e parasitárias: guia de bolso / Ministério da Saúde, Secretaria de Vigilância em Saúde, Departamento de Vigilância Epidemiológica.- Brasília: Ministério da Saúde: 2010 - - 8. ed. rev.
24. Ministério da Saúde (BR). Acidentes por animais peçonhentos - Brasília: Ministério da Saúde: 2016 -[citado em 25 mar. 2017]. Disponível em: http:// portalarquivos.saude.gov.br/images/pdf/2016/maio/20/ Informe- Epidemiol--gico-animais-pe--onhentos---.pdf

25. Ministério da Saúde (BR). Serpentes. Brasília: Ministério da Saúde: 2016 - [citado em 03 de mar. 2017]. Disponível em: htps//portalsaude.saude.gov.br/index. php/o- ministerio/principal/secretarias/svs/animaispeconhentos-serpentes

26. Moreno, E, Andrade, MQ, Lira, SRM - Características clínicoepidemiológicas dos acidentes ofídicos em Rio Branco - AC, 2008- Revista da sociedade Brasileira de Medicina tropical, vol. 38, São Paulo,7p

27. Moura, GM. Tudo sobre escorpião. Brasília: Ministério da Saúde: 2015 - [citado em 17 jan. 2017] - Disponível em: http://minhamemoria-gleison.blogspot.com.br/2015/07/ tudo-sobre- escorpiao.html

28. Moura, V. Período chuvoso favorece ocorrência de acidentes com animais peçonhentos; aranhas e cobras são os mais comuns em Rondônia, NoticiasPortal do governo do estado, secretaria de estado do desenvolvimento ambiental. Rondônia: 2016- [citado em 20 dez. 2017]. Disponível em: http://www.rondonia. ro.gov.br/2016/11/145507

29. Notícias- Enchentes. Cacoal: 2012 - [citado em 01 abr. 2017]. Disponível em: https://www.ariquemesonline. com.br/noticia.asp? cod $=253597 \& \operatorname{codDep}=31$

30. Oliveira, HFA, Costa, CF., Sassi, R. Relatos de acidentes por animais peçonhentos e medicina popular, Paraíba: 2013 - [artigo]. Brasil -Rev Bras Epidemiol 2013; 16(3): $633-43$

31. Pena, RFA. - Enchentes - Mundo Educação - 2014 - [citado em 23 mar. 2017]. Disponível em: http:// mundoeducacao.bol.uol.com.br/geografia/enchentes. htm

32. Pereira, ID, Pinho, FMO. Ofidismo - São Paulo: 2001 Rev. Assoc. Méd. Bra. São Paulo, v. 47, n. 1, p. 24-29

33. Pinho, FMO, Pereira, ID. Ofidismo. Rev. Assoc. Med. Bras. [online]. 2001, - [internet] - Disponivel em: <http:// dx.doi.org/10.1590/S0104- 42302001000100026. vol.47, n.1, pp.24-29. ISSN 0104-4230

34. Prefeitura municipal de Cacoal - Dados secundários/ animais peçonhentos - Prefeitura municipal de Cacoal: Secretária municipal de saúde: vigilância em saúde de Cacoal

35. Projeto Estudo Genético de Tityus obscurus, UFPA- PA: 2010 -[citado em 05 abr. 2017]. Disponível em: http:// lemap-ufpa.webnode.com/projeto-estudo-genetico-detityus- obscurus 
36. Silva, JJB ; Gomes, FBC, Cezário, AC, Moura, L. - Doenças e agravos não transmissíveis: bases epidemiológicas. In: Rouquayrol MZ, Almeida Filho. Epidemiologia \& Saúde. 6a ed. Rio de Janeiro- RJ -2003: Medsi;.. p.289-311. 8. Organização Mundial da Saúde. Manual de classificação internacional de doenças

37. Yamaguchi, IK. Soro antibotrópico. São Paulo: 2012 [citado em 23 de fev. 2017]-Disponível em: http://www.butantan. gov.br/Documents/soro-antibotr\%C3\%B3picopentavalente.pdf

\section{Como citar este artigo:}

Faria G, Lima AAM. Aspectos epidemiológicos dos acidentes por animais peçonhentos ocorridos no município de Cacoal-RO, no período de 2007-2016. Rev. Aten. Saúde. 2019; 17(61): 45-56. 